\title{
I BAMBINI E NOI: L'INFANZIA NEL CINEMA DI LUIGI COMENCINI
}

\section{Giovanna De LuCA}

\begin{abstract}
Riassunto: Padre per antonomasia dei bambini del cinema italiano, Luigi Comencini ha dedicato tredici film all'infanzia ripercorrendo l'evoluzione storico sociale del paese dal secondo dopoguerra fino agli anni 90. Questo saggio si propone di investigare, attraverso l'analisi di alcuni dei film più esemplari del regista, due temi ricorrenti del discorso comenciniano sull'infanzia: la coesistenza nel bambino di una dipendenza dall'adulto e un desiderio di autonomia e l'esplorazione dell'ambiguo rapporto padre/figlio.
\end{abstract}

L'eclettico Luigi Comencini, regista-architetto italiano-francese, ha attraversato la storia del cinema italiano camaleonticamente, rappresentando con sguardo semplice e razionale la condizione umana soprattutto attraverso i bambini, espressione artificiosa di quella umanità che era desideroso di raccontare. Spaziando tra cinema-documentario, neorealismo rosa, commedia all'italiana e cinema dell'infanzia, Comencini è stato il regista italiano testimone delle diverse fasi e tendenze del cinema nazionale, ripercorrendo l'evoluzione del Paese dal secondo dopoguerra fino agli anni 90. Il tema dell'infanzia in particolare ha un posizione rilevante nella copiosa produzione artistica del regista. Tra documentari, film televisivi e film per il grande schermo annotiamo: I bambini in città (1946), Heidi (1952), La Finestra sul luna park (1956), Incompreso (1967), Infanzia, vocazione e prime esperienze di Giacomo Casanova (1969), I bambini e noi (1970), Le avventure di Pinocchio (1972), Voltati Eugenio (1980), Cuore (1984), La storia (1986), Un ragazzo di Calabria (1987), Tutti i bambini ... di Comencini (1990), Marcellino, (1991). Il suo penultimo film, Tutti $i$ bambini ... di Comencini, è un omaggio a Comencini in quanto padre per antonomasia dei bambini del cinema italiano, e un omaggio del regista a tutti i bambini che hanno informato le sue opere dedicate ai bambini. Trasmesso dalla RAI in 5 puntate, il film-documentario riflette sull'infanzia portata sugli schermi in 50 anni della sua carriera.

Tra i diversi episodi, emblematico della sua visione temporale dell'infanzia è Ricordi e segreti del mestiere, in cui il regista spiega come è nata l'idea di rivisitare i bambini del suo cinema diventati adulti' ${ }^{1}$ :

1 Perchè molto provato fisicamente, per questo film Comencini si è avvalso dell'aiuto del suo collaboratore Giulio Manfredonia. 
Mi venne in mente di far cercare tutti i bambini con cui avevo lavorato. $\mathrm{Li}$ feci riprendere, a loro insaputa, dalla macchina da presa. Entravano uno alla volta e si guardavano ignari, senza conoscersi. Li osservavo non visto. Io li riconoscevo tutti anche molto cambiati. Erano gli stessi ma loro non c'erano più ${ }^{2}$.

La fanciullezza come limitato periodo dell'umana esistenza, e la libertà dai condizionamenti degli adulti in questo circoscritto spazio temporale, sembrano essere alla base dell'interesse di Comencini per questo argomento. Alla domanda postagli da Lorenzo Codelli riguardo il ritorno al tema dell'infanzia nel film La Finestra sul luna park, Luigi Comencini risponde:

Evidentemente è la mia predilizione costante. Non so perché, non è un programma che mi sia prefisso ... prova ne sia che quando cerco di rappresentare un mondo nuovo, interamente mio, ci ricasco sempre. ... Infatti anche per Infanzia, vocazione e prime esperienze di Giacomo Casanova veneziano ho scelto Casanova bambino, e non so perchè. Mi sembra che l'infanzia abbia una caratteristica fondamentale, di essere il solo periodo di grande libertà dell'individuo. Il processo attraverso cui l'educazione scolastica e familiare, tende a soffocare questa libertà è drammatico. ... Il solo mezzo per liberare l'infanzia è proprio mettersi al suo livello ${ }^{3}$.

Sebbene questa affermazione suggerisca che i riccorrenti incontri sullo schermo tra Comencini e l'infanzia siano di natura casuale, in realtà sia il vissuto familiare del regista (che lo ha portato a vivere soprattutto con donne), sia scelte stilistiche e di produzione (soprattutto televisive, favorevoli ad una familiare proiezione cinematografica infantile, in un periodo di forti scontri ideologici sul piano nazionale), sono alla base dell'attenzione del regista per la fanciullezza. Il risultato è un cinema etico sempre attento agli individui più deboli della società, in particolare donne e bambini. Come per altri registi che si sono dedicati all'infanzia, i bambini sono spesso un tramite, cartine al tornasole per far luce sugli adulti, affinché questi ultimi possano comprendere come recuperare, attraverso la visione dell'infanzia, la propria infanzia come momento di libertà assoluto. Quando Comencini suggerisce di "mettersi a livello dell'infanzia" per poterla liberare dalla sovrastruttura educativo-morale creata dall'adulto, egli propone l'adozione di un'osservazione del reale senza filtri e a 360 gradi, come quella dei bambini. L'infanzia diventa in questo modo anche strumento di

2 Adriano Aprà, Luigi Comencini: Cinema e film, 51.

3 Luigi Comencini Il cinema secondo me. Scritti e interviste (1974-1992), 23. 
apprendimento e consapevolezza di se stesso e dell'altro, un espediente costruttivo per l'interazione con l'altro.

Nel suo cinema dell'infanzia Comencini usa il topos del genere Bildungsroman e alterna il racconto favolistico alla storia avvalendosi spesso dell'alternanza tempo determinato/tempo ricreato ${ }^{4}$. Questa binaria esposizione dell'infanzia, che si distacca da quella politico-sociale neorealista, ha permesso al regista di comunicare con un pubblico adulto (soprattutto con il canale televisivo) usando quell'alternanza favola/ realtà caratteristica dei bambini, alla quale l'adulto riesce a rapportarsi in quanto richiamo al proprio vissuto senza un apparente appello alle sue responsabilità. Nell'alternata presentazione di apparenza e sostanza, immaginario e realtà, si colloca l'invito comenciniano a guardare il mondo come i bambini.

Sia nei film televisivi (documentari e serie che a detta del regista gli "offrivano più libertà creativa perché meno preoccupato della reazione del pubblico") che nei film per il grande schermo, Comencini ha dimostrato di non essere mai impreparato a fotografare i suoi tempi, e nel rispetto delle sue scelte etiche, a dare un contributo alla creazione di un' Italia migliore. Nel 1946, quando con Sciuscià, Zavattini e De Sica rendono noto al pubblico italiano e straniero come il cinema possa contribuire alla ricostruzione morale e civile dell'Italia, Comencini gira il suo primo film, il documentario Bambini in città, ambientato nella Milano nell'immediato dopoguerra. Il film offre uno spaccato di vite mutilate dall'avvento della guerra. La camera si poggia sulle macerie delle città distrutte dove vivono donne che hanno affrontano da sole lutti familiari, e bambini orfani, testimoni della distruzione fisica e morale del Paese. Essi sono però allo stesso tempo portatori di una disposizione "libertaria" che li conduce a sperare di abitare/esperire altri spazi 5 . In questo primo film sono già in nuce i rimandi tematici comuni a tutta la produzione cinematografica di Comencini dedicata all'infanzia: la coesistenza nel bambino di una necessità di dipendenza e distacco dall'adulto e l'esplorazione del rapporto padre/figlio. Il regista propone sullo schermo con realistica chiarezza questa duplicità infantile: la distaccata rappresentazione di un'esistenza "between," a metà tra dipendenza dall'adulto e desiderio di indipendenza e autosufficienza. Il critico francese Jean Gili così interpreta questo aspetto:

Comencini si è impegnato a mostrare la profonda dualità del bambino: la sua dipendenza e la sua autonomia. Il bambino ha avuto a volte bisogno del-

4 Giovanna De Luca, Il punto di vista dell'infanzia nel cinema italiano e francese: rivisioni, 99.

5 Aprà, Luigi Comencini: Cinema e film, 43. 
l'adulto, del padre e della madre, ed è allo stesso tempo portatore di un'indipendenza vitale, di una capacità di affrontare la vita intuitivamente senza l'apprendistato al quale vogliono sistematicamente sottometterlo gli adulti ${ }^{6}$.

Rientrano così in questa tipologia personaggi reali quali i bambini napoletani di otto/nove anni dell'episodio La fatica del documentario I bambini e noi. I giovani protagonisti vengono forzati dalle condizioni economiche indigenti delle proprie famiglie a lavorare in cocenti vetrerie o nei sotterranei di fabbriche di scarpe. Con la dignità di un adulto un ragazzino nasconde all'intervistatore, lo stesso Comencini, di aver perso il pollice di una mano con una sega elettrica al lavoro, nel timore di perdere, denunciando involontariamente il suo datore di lavoro, l'unica fonte di denaro per sé e la sua famiglia. Con la stessa compostezza, il piccolo garzone del bar minimizza lo stato di miseria che lo porta a mangiare una mela perché non ha soldi per il pranzo. La loro adulta riservatezza è accompagnata anche da un malcelato desiderio di protezione e appartenenza familiare, anche se a causa di quella stessa famiglia sono costretti a "faticare" sin da piccoli. L'elogio dell'indipendenza fanciullesca e adulta è identificabile anche nel personaggio di Casanova in Infanzia, vocazione e prime esperienze di Giacomo Casanova. Da piccolo Giacomo è un bambino spesso solo, con una madre attrice assente e un padre ammalato che prematuramente morirà. La nonna è l'unica che, con interesse, si prende cura di lui. Quando anche la nonna viene a mancare, il piccolo Giacomo comincia a ribellarsi a questa privazione affettiva. Prima di essere mandato a studiare a Padova alla scuola di Don Gozzi, viene rinchiuso per la sua condotta turbolenta nella fortezza Forte di Sant'Andrea. La prigionia preventiva rafforza la sua autonomia senza smussarne il carattere ribelle che alla fine lo porterà al successo.

Il giovane Eugenio in Voltati Eugenio diventa per Comencini l'allarmante lume della confusione ideologica della società sessantottina, della crisi dei valori borghesi e i loro effetti sulla generazione dei figli. Eugenio, figlio di una coppia troppo coinvolta nell'esplorazione di nuove avventure ideologiche per preoccuparsi tradizionalmente di lui, viene sballottato continuamente tra diverse case di genitori e nonni, fino a quando decide di lasciarsi dietro il confuso nucleo familiare e incamminarsi da solo, finalmente libero, per il suo incerto destino.

6 Jean Gili, Luigi Comencini, 89. "Comencini s'est attachè à montrer la profond dualité de l'enfant: sa dépendance et son autonomie. L'enfant a à la fois besoin de l'adulte, du pére et de la mére, et est en même temps porteur d'une indépendence vitale, d'une capacié d'affronter la vie de maniére intuitive sans l'apprentissage auquel veulent systématiquement les soumettre les adulte". 
Lo stesso si dica per il piccolo Useppe, il giovane protagonista di La storia, adattamento televisivo/cinematografico del romanzo di Elsa Morante. Ambientato a Roma durante la seconda guerra mondiale, il film segue le vicissitudini di Ida Ramundo, vedova Mancuso, giovane maestrina metà ebrea ed i suoi figli Nino e Useppe. Quest'ultimo è frutto di una violenza subita da Ida da parte di un giovane soldato tedesco, atto che lascerà un segno indelebile nelle esistenze di madre e figlio. Come suo fratello Nino, anche il piccolo Useppe morirà, evento che porterà Ida alla follia. La fragile Ida durante la gravidanza e nei primi anni di vita aveva ingenuamente cercato di tenere nascosta l'illegittima nascita del piccolo. Useppe sin dai primi giorni della sua breve esistenza, ha dovuto fare i conti con un'indipendenza dapprima forzatamente imposta (quando la madre lo lasciava solo per andare a scuola) trasformatasi a poco a poco in una modus vivendi che ha delineato il carattere del ragazzino. Il piccolo Useppe durante il periodo dello sfollamento nella grande casa comune, riesce da solo a comunicare con tutti e a stabilire rapporti di autonoma amicizia con adulti, piccoli e animali.

Il Pinocchio comenciniano è forse il personaggio che meglio incarna la vita infantile tra dipendenza e autonomia. Nell'adattamento televisivo di Comencini, il Pinocchio collodiano diventa subito bambino con tutte le reazioni e i desideri dell'infanzia. Viene trasformato in burattino ogni qual volta disobbedisce a quelle che sono le norme del vivere comune imposte da Geppetto e dalla madre/Fata Turchina (altra modifica alla stesura collodiana). La metafora del burattino creato dal falegname Geppetto, simbolicamente riproduce le fasi della crescita infantile dallo status neonatale dell'infante, alla sua trasformazione in un bambino in carne ed ossa, alla crescita e con essa la presa di coscienza della sua autonomia e libertà. La dualità burattino-dipendente e bambino-autonomo, che nel capolavoro collodiano si risolve con l'assunzione di sembianze umane solo alla fine del racconto quando il burattino si è conquistato l'umanità, nell'opera comenciniana viene presentata sin dall'inizio con l'invenzione di un personaggio unico, con una "sola anima e un solo carattere" 7 . Personaggio fiabesco e di costume, Pinocchio tuttavia si avvicina ai soggetti realisti dei documentari comenciniani proprio nella sua metaforica dualità di burattino/bambino. Pinocchio, come qualsiasi bambino, si dimostra affettuoso e bisognoso di cure ma allo stesso tempo è uno spirito libero e insofferente alle regole. Istintivamente rifiuta l'ubbidienza a discipline morali dettate dal conformismo degli adulti. Questa visione viene rinforzata anche dalla intenzionale rimozione dal film di personaggi etici come il grillo parlante, che Comencini riduce ad una semplice silhouette, o

${ }^{7}$ Adriano Aprà, Comencini:Cinema e film, 184. 
dall'ammortizzazione dell'aura presenza della Fata, la matrigna/fantasma, che nel film diventa "strega" agli occhi di Geppetto, quando le rimprovera l'applicazione di suoi sistemi educativi ricattatori nei confronti di Pinocchio. Come per il personaggio collodiano, il Pinocchio di Comencini è l'antieroe "ribelle mancato" secondo l'interpretazione che Martin Jarves dà al personaggio di Collodi, ma non corrisponde però al "perpetuamente bambino mancato" individuato dallo stesso Jarvis nel personaggio letterario ${ }^{8}$. La fisicità infantile del Pinocchio comenciniano è immediatamnte enfatizzata e si manifesta attraverso corse spericolate, parole urlate, e forti attacchi di fame. Allo stesso tempo il regista dà al suo Pinocchio una forte sensibilità infantile in relazione al suo status di orfano (di madre) ed il suo legame col padre, che il regista ritrae in maniera molto più realista del nel libro. Pinocchio è nel film di Comencini un bambino riuscito a tutto tondo.

Al discorso comenciniano sull'esperienza infantile del duplice desiderio di autonomia e dipendenza, va aggiunta come altra ricorrenza tematica nel cinema di Comencini, la conflittuale relazione padre-figlio, o quella del bambino simbolicamente orfano di genitori. Lo stesso Comencini afferma al riguardo:

Questa fissazione sui rapporti tra padre e figlio si spiega forse con il fatto che non ho avuto dei veri rapporti con mio padre: è morto quando avevo 18 anni e il rapporto con i bambini era 'monopolizzato' da mia madre. Mio padre era un personaggio misterioso, andava sempre in giro per casa; mi sarebbe piaciuto avere con lui un contatto, ma non mi riusciva di stabilirlo?.

La lettura sociale della condizione orfana del bambino riflette un cambiamento antropologico legato alla modernità e in Occidente in particolare, alla rivoluzione culturale sessantottina. Riprendendo l'analisi di Giacomo Debenedetti, con la morte del padre, della freudiana possibilità di colpevolizzarlo per il male di vivere dei figli, alle giovani generazioni non resta che fare di tale "orfanezza e degli squallori e sgomenti di questa solitudine la nuova condizione umana" 10 . Nell'ambito di questo nuovo status ontologico, il personaggio infantile viene ritratto alla continua ricerca di una figura paterna che alla fine non viene mai trovata. Ma Comencini sembra suggerire che proprio nell'atto della ricerca e non nel ritrovamento si esaurisce e completa la nuova condizione di orfano ${ }^{11}$.

8 Prefazione a Carlo Collodi, Le avventure di Pinoccho. Storia di un burattino. (Torino:Einaudi 2002), XXXI-XXXII.

9 Giorgio Gosetti, Luigi Comencini, 4.

10 Giacomo Debenedetti, 118

${ }^{11}$ De Luca, Il punto di vista dell'infanzia nel cinema italiano e francese: rivisioni, 100. 
L'universo infantile di Comencini è caratterizzato da padri assenti, incapaci o scomparsi e madri non proprio ineccepibili. In La finestra sul luna park, nella periferia romana, tra le umili dimore di borgata, Comencini, attento lettore della società contemporanea, riflette sul problema dell'emigrazione, non solo per coloro che partono in cerca di fortuna, ma anche per chi rimane in un'economia arretrata e soprattutto in una situazione monca di affetti. Mario, un ragazzino di otto anni rimasto orfano di madre (morta in un incidente), non riesce a stabilire un rapporto affettivo con suo padre, Aldo, emigrato in Kenya all'inseguimento del sogno piccolo borghese. Al vero padre, Mario preferisce il padre adottivo Righetto, l'umile borgataro che si è sostituito ad Aldo durante la sua assenza e che alla fine riconcilierà l'unione tra padre figlio. Nato sulle ceneri del Neorealismo (la cura con cui vengono esposti ambienti degradati dove ha luogo l'azione, la comunicazione dialettale degli umili sottolineano una denuncia sociale di problemi contemporanei scottanti quali la disoccupazione e l'emigrazione), il film tuttavia ambisce al raggiungimento di un pubblico più ampio, tralasciando il j'accuse politico ed entrando invece nella sfera dei sentimenti, in contrapposizione agli interessi materiali ed economici. Nel film di Comencini la figura paterna ha perso l'aura patriarcale, conservando solo l'autorità e la caparbietà di inseguire il sogno borghese. Aldo vuole "pensare al domani, solo al domani" senza aperture agli affetti che potrebbero intralciare il suo piano. Comencini, in una fase ancora sperimentale da inizio carriera, investe nel sentimento e nella presa di coscienza del genitore la realizzazione di un cambiamento nel rapporto padre-figlio. Questa visione verrà poi abbandonata gradualmente durante la sua carriera per dare spazio ad una percezione più nichilista, che vede nello stato ontologico di orfano l'ultima scelta possibile per il bambino. Solo con il suo penultimo film dedicato all'infanzia, Un ragazzo di Calabria, il regista riconsidererà il suo approccio iniziale, che vedeva nell'adulto la maggiore responsabilità del cambiamento nella ricostruzione dello strappo affettivo-cognitivo tra padri e figli.

L'ultima scena del film La finestra sul luna park è un richiamo al famoso finale di Ladri di biciclette. Mario e Aldo finalmente si ricongiungono in prossimità del luna park vicino a casa. Mettendo da parte il chiuso dispotismo che lo ha finora caratterizzato, il padre confida al figlio di "aver paura" dei seggiolini volanti. Un gesto di maturità che si inserisce in quella universale, riconoscibile scala di valori cara a Comencini ed ai suoi spettatori.

Anche Milo in Incompreso, il film strappalacrime adattamento cinematografico dal famoso romanzo di Florence Montgomery Misunderstood del 1869 , indica che la complessa relazione padre-figlio trascende le problematiche del proletariato romano e si estende anche all'alta borghesia. Nell'adat- 
tamento cinematografico Comencini mantiene la nazionalità originale inglese della famiglia, ma la trasferisce sulle colline fiorentine. In una villa isolata dal mondo vivono il console inglese John Edward Duncombe e i suoi due figli, il primogenito Andrea e il secondogenito Milo. Il dramma della morte della madre e come esso abbia influito nell'intricata relazione padre-primogenito, viene qui diluito nella creazione di un rapporto delicato e competitivo tra i due fratelli e nella rappresentazione di un'elaborazione del lutto vissuta molto privatamente sia da Andrea che dal padre. La giovane età invece ha permesso a Milo di vivere l'esperienza del lutto in maniera più positiva, rifugiandosi in un fantasmatico mondo di madri defunte presenti comunque nel quotidiano della propria esistenza. Comencini vede la malvagità di Milo, il piccolo bugiardo che provoca indirettamente la morte del fratello, come una fase naturale dell'esitenza infantile. Una sorta di meccanismo di autodifesa attraverso il quale il ragazzino protegge se stesso dal mondo forse ancora più malvagio degli adulti.

Duncombe si trova ad affrontare la mancanza fisica della moglie-madre al cospetto di due diverse sensibilità infantili che è incapace di comprendere. Fermandosi a una superficiale interpretazione delle reazioni ribelli del figlio Andrea (lo scarso profitto scolastico, il burrascoso rapporto con la governante e quello antagonistico con il fratello minore), risponde al suo comportamento con un'indifferenza emotiva che porterà alla fine alla morte del figlio. Regista interessato dei sentimenti piuttosto che ai sentimentalismi, Comencini neutralizza la componente melodrammatico-patetica del romanzo rifuggendo il banale artificio della presa sull'emotività dello spettatore e mettendo al centro dell'intreccio narrativo il punto di vista infantile: la scoperta della diversa "sensibilità del mondo degli adulti" nei confronti della morte di un genitore ${ }^{12}$. Propone quindi scene slegate che non seguono necessariamente la logica narrativa del romanzo, ma piuttosto quella del racconto filmico che procede per ellissi e sottrazioni, dove l'incombenza del fantasma della morte viene suggerita soprattuto attraverso lo scavo psicologico della reazione dei bambini alla scomparsa della madre. Il vuoto emotivo che separa i membri della famiglia Duncombe e coloro che sono direttamente o indirettamente coinvolti nella loro particolare situazione (lo zio Willy, la governante Dora, ecc.) viene esibito tramite ambienti vuoti e rigidi che favoriscono la distanza fisica tra personaggi. Solo nel finale, quando Andrea è agonizzante e il padre è al suo capezzale, in un gioco di specchi tra padre, figlio e ritratto della madre osservato da Andrea, avviene finalmente la riconciliazione emotiva ed affettiva.

12 Aprà, Luigi Comencini: Il Cinema e i film, 172. 
Con La storia Comencini inizia un graduale riposizionamento della figura dell'orfano nella sua opera, che da incompreso si trasforma in portatore di verità assoluta. Rispettando l'epitaffio d'apertura del romanzo della Morante, il regista ricorda come attraverso innocenza, malattia e morte, il giovane protagonista Useppe sia uno dei piccoli a cui Dio ha rivelato la verità. Lo status di orfano di Useppe in questo film è aggravato dalla componente violenta legata al suo concepimento e dalla particolare natura di Ida, donna-bambina che attraversa la maternità e la guerra con timore ed inerzia. Anche in questo caso, come per l'adattamento di Incompreso, Comencini ed i suoi co-sceneggiatori (la figlia Cristina Comencini e Suso Cecchi D’Amico) riescono a smorzare i toni melodrammatici del romanzo morantiano sostituendo il punto di vista del narratore onnisciente femminile con quello del piccolo Useppe, un semplice ragazzino. L'alterità dello sguardo infantile-altro, perché posto al confronto con quello razionale dell'adulto, permette a Comencini di osservare l'ingombrante storia che Morante vede imposta dai potenti sulle masse con occhi integri dell'infanzia. Senza il bagaglio dell'esperienza dell'adulto, Useppe vede la guerra ponendo le componenti crudeltà e umanità su uno stesso livello percettivo. Questo cambio prospettico facilita un'indentificazione emotiva con il narratore bambino, in opposizione a quella cognitiva col narratore onnisciente.

Nel romanzo la statura mitica del bambino viene stabilita attraverso la premonizione della sua morte suggerita enfatizzando la sua sua unicità, innocenza e apertura verso gli altri. Il film di Comencini rispetta alcuni tratti caratteriali di Useppe usati nel romanzo, quali innocenza e simbiotico rapporto con natura e animali, ma nel film il bambino si distingue soprattutto per vivacità e curiosità, qualità che non lo rendono dissimile da altri bambini. Come in tutte le morti infantili letterarie e cinematografiche, nel romanzo la morte di Useppe ha una funzione espiatoria. Nel film essa si consuma quasi naturalmente senza grandi premonizioni. Useppe da solo, con il suo cane Bella alla ricerca del suo amico Scimò, soccombe all'ultimo attacco epilettico. In questa prospettiva naturalista la morte di Useppe acquista il valore di atto di ribellione e protesta per le vittime della storia.

La svolta definitiva alla problematicità delle relazioni padre/ figlio orfano, adulti/bambini viene proposta in Voltati Eugenio. Il film ha le sue radici nelle inchieste televisive degli anni settanta quali I bambini e noi e L'amore in Italia, i cui intervistati (rispettivamente il giovane Luca Rolla, il giovane malinconico poeta e una coppia sessantottina) hanno ispirato la creazione del personaggio di Eugenio e dei suoi giovani genitori Fernanda e Giancarlo.

Comencini provocatoriamente apre il film con uno spintone. Eugenio viene letteralmente gettato fuori dall'auto da Baffo, redattore di un giornale satirico che, infastidito dalle pungenti domande del ragazzo, lo abban- 
dona in una strada di campagna. Questa scena iniziale, immagine metaforico-familiare, presagisce lo sviluppo narrativo del film e soprattutto il suo finale. Vivendo una situazione familiare dove nessuno vuole assumersi la responsabilità della sua crescita, Eugenio viene esortato a diventare indipendente a crearsi una propria vita. Partendo dall'espulsione dall'auto e dalla scomparsa del ragazzino, il film copre una giornata di ricerche da parte della famiglia e polizia. La narrazione si sviluppa attraverso una serie di flashback che ripercorrono la vita del ragazzo dal suo concepimento fino al giorno della sparizione. Eugenio viene ritrovato dopo un giorno di ricerche in una stalla dove si era fermato per assistere alla nascita di un puledrino. Dopo il ritrovamento, nonni e genitori ricominciano a litigare su chi deve prendersi la responsabilità di ospitarlo. A questo punto Eugenio lascia la stalla senza che nessuno se ne accorga, sollecitato anche da Baffo, che ancora una volta dimostra di essere il meno ipocrita tra i grandi. L'uscita di scena di Eugenio e del suo cane, che si allontanano dalla stalla, inghiottiti da un potente fascio di luce, sottolinea la dimensione favolistica che il regista ha voluto dare al film. Questo approccio gli ha permesso di dare una funzione didattico-didascalica al racconto.

Descrivere la storia con la favola diventa allora l'escamotage organizzativo ideale su cui impiantare un film-patchwork fatto di frammenti di storia c'è uno spazio qui di personaggi e ideologia, dove prevale un'esposizione distanziata, non dialettica e soprattutto irrisolta della recente storia. Privilegiando la descrizione di stati d'animo rispetto agli eventi che li hanno scaturiti, Comencini propone una riflessione sulle compromesse interazioni umane piuttosto che una critica visione del mondo. Il motore che mette in funzione il suo cinema è legato alle emozioni che precedono sempre l'ideologia asservita ad essi 13 .

Nonostante Eugenio sia vittima di un'ideologia incerta, raramente viene rappresentato come tale. $\grave{E}$ un ragazzo perspicace, pronto a ribattere e mettere in discussione con sarcasmo e senza peli sulla lingua le vaghe giustificazioni comportamentali dei membri della propria famiglia. Per passare all'azione, per trovare la sua strada, gli manca solo una sorta di autoritario incitamento (autoritario perché gli viene dall'adulto Baffo).

Giancarlo, la figura paterna in questo film, è immaturo ed egoista ma soprattutto ipocrita. In un mondo di ambiguità e incoerenze, l'unico portatore di verità è il sarcastico Baffo, l'outsider, il buffone sbrigliato da qualsiasi convenzione sociale, anche rivoluzionaria, a cui Comencini affida il ruolo di ribaltatore dello status quo. Baffo è il fool a cui è concesso rivelare il contrario

13 Giovanna De Luca, "Fable and Reality in Comencini’s Voltati Eugenio" 214. 
di tutto (secondo l'accezione bakhtiniana) e quindi la verità. Come gli altri alter-ego paterni del cinema comenciniano (mi riferisco a Richetto ne $\mathrm{La}$ finestra sul luna park e Felice in Un ragazzo di Calabria) Baffo si rivela pur nella sua cruda brutalità l'unico personaggio capace di riconoscere l'esistenza di Eugenio. Rivelandogli la verità sui suoi rapporti parentali, gli fornisce l'unica alternativa a tanta indifferenza. La decisione di Eugenio di abbadonare completamente la sua famiglia si può leggere in termini di fuga secondo l'accezione fornita dal bio-sociologo Henry Laborit. Per Laborit il comportamento della fuga è necessario all'individuo per poter preservare il suo benessere biologico nella battaglia continua in seno a oppressive gerarchizzazioni e imposizioni sociali14. A Eugenio sin dalla nascita non è stato riconosciuto né lo status di bambino, né quello di figlio. Alla ricerca di una legittimazione come soggetto, la fuga dal disinteressato nucleo familiare è l'unica alternativa per l'acquisizione della libertà ed identità.

Lapproccio nichilista di Voltati Eugenio cede il posto ad una visione più riconciliante dello scarto padre-figlio nel film Un ragazzo di Calabria. Comencini riprende la tematica del padre-padrone e della doppia paternità, biologica e affettiva, affrontata ne la La finestra sul luna park e la trasferisce a Sud.

La storia si svolgeva nel 1960, anno delle Olimpiadi di Roma. L'idolo del protagonista Mimì è Abebe Bikila, l'atleta etiope che vincendo la maratona concluse con un trionfo africano le Olimpiadi. Una volta tanto un ragazzo del Sud non guarda verso il Nord, ma vuole imitare l'impresa di un campione africano. In questo suo sogno è ostacolato dal padre, Diego Abatantuono, e incoraggiato da un autista sciancato suo amico, Gian Maria Volontè ${ }^{15}$.

Nicola è un padre violento che lavora in un manicomio come uomo delle pulizie grazie a un parente colluso con la mafia. Lo stesso parente dovrebbe trovare un posto di lavoro anche a suo figlio Mimì quando questi terminerà gli studi. Nicola è un uomo che vive ai limiti della società $\mathrm{e}$ che ha riposto speranze di una vita migliore interamente nel figlio. È incomprensibile per lui che Mimì abbia una passione per un'attività non pratica o comunque non remunerativa a lungo termine. La sua violenza verso il figlio si può intrepretare come gesto di impotenza e paura nei confronti di una libertà di scelta ed azione che lui non ha mai avuto. Anche qui come ne La finestra sul luna park, il padre putativo, Felice, è il 'padre per scelta' che il giovane Mimì sceglie. Il segno che contraddistingue que-

14 Henry Laborit, Elogio della fuga.

15 Luigi Comencini. Infanzia, vocazione di un regista 147. 
sto genitore è proprio la sua diversità, sia fisica (ha un handicap che gli procura il soprannome "lo sciancato"), che ideologica (di estrema sinistra) alla base di un'esistenza marginale nella retrograda società meridionale. Un personaggio liminale dunque, condivide con Nicola una simile emarginazione sociale. Film meno intimista di Incompreso, Un ragazzo di Calabria rivede l'emarginazione socio-geografica del Sud italiano attraverso gli occhi di un ragazzino che, a dispetto di tutte le aspettative, ne esce vincente, riscattando brevemente la subalternità storica della sua terra. La rievocazione da parte di Felice della maratona di Filippide a Mimì, ben sottolinea il messaggio di emancipazione proposto da Comencini: "La gara per i disgraziati come noi, abituati a faticare sin dalla nascita. È la tua gara, Mimì. È la gara che devi vincere. Tu, quando corri, corri sempre lunghe, lunghe distanze". In questa storia di emarginazione le paternità biologiche e affettive sono riscattate dalla passione e dalla ribellione di un giovane ragazzo che è riuscito ad osare di più di loro. Anche qui, alla paternità deficiente Comencini affianca una paternità meno autoritaria, quasi fratellevole. Sebbene il film sia stato girato nel 1987 quando oramai era chiaro che l'auspicata ripresa ed integrazione del Sud non sarebbero mai avvenute, termina con una nota di apertura che incoraggia a sperare/sognare in un cambiamento, esattamente come fa Mimì quando corre. Con Un ragazzo di Calabria Comencini si congeda in maniera riconciliata dal complesso discorso sul rapporto padre-figlio centrale alla sua cinematografia. L'immagine vincente di Mimì racchiude in sè tutte le immagini delle infanzie violate ma sopravvissute grazie alla resilienza insita nell'infanzia stessa.

\section{College of Charleston}

\section{Opere citate}

Aprà, Adriano Ed. Luigi Comencini. Il Cinema secondo me. scritti e interviste (19741992). Milano: Il Castoro, 2008.

—. Luigi Comencini: Cinema e film. Venezia: Marsilio, 2007.

Benayoun, Robert. "Luigi Comencini." Positif 156 (1974): 56-58.

Cecconi, Luciano. I bambini nel cinema, Milano: Franco Angeli, 2006.

Collodi, Carlo. Le avventure di Pinocchio. Storia di un burattino (Torino: Einaudi, 2002)

Comencini, Luigi. Infanzia, vocazione, esperienze di un regista. Milano: Baldini e Castoldi, 1999.

Il cinema secondo me. Scritti e interviste (1974-1992) Milano: Il castoro, 2008.

Contini, Gabriella. "Useppe." "Venti anni dopo 'La Storia' Omaggio a Elsa Morante." Concetta d'Angeli and Giacomo Magrini, eds. Studi novecenteschi 21. 47-48. Pisa: Giardini Edition, 1994. 185-213. 
Debenedetti, Giacomo. Personaggio-uomo. Milano: Garzanti, 1998.De Luca, Giovanna. Il punto di vista dell'infanzia nel cinema italiano e francese: rivisioni. Napoli: Liguori Editore, 2009.

"Fable and Reality in Comencini's Voltati Eugenio," Italica, 82. 2 (2006): 210-221

Gili, Jean. Luigi Comencini. Paris: Edilig, 1980.

Gosetti, Giorgio. Luigi Comencini. Firenze: La Nuova Italia, 1989.

Kuhn, Reinhard. Corruption in Paradise: The Child in Western Literature. Boston: Boston UP, 1982.

Laborit, Henry. Elogio della fuga. Milano: Mondadori, 1982.

Lury, Karen. The Child in Film: Tears, Films and Fairy Tales. London: IB Tauris and Co. Ltd., 2010.

Toubiana, Serge. "Les Enfants et nous.” Cahiérs du cinéma 143 (1981): 60-61 